\title{
Influence of Perceived Organizational Support on Employees' Affective Commitment in Railway Enterprises: From the Mediating Role of Overconfidence
}

\author{
Hongxia Tong ${ }^{1,2, a}$, Shunhong Wang 1, b \\ ${ }^{1}$ School of Economics and Management of Southwest Jiaotong University, Chengdu 610031, \\ China
}

${ }^{2}$ Shanghai Research Institute of Southwest Jiaotong University, shanghai 200072, China

atonghongxia@swjtu.edu.cn, bwang413@home.swjtu.edu.cn

\begin{abstract}
Keywords: High speed railway, perceived organizational support, overconfidence, affective commitment, mediating role.
\end{abstract}

\begin{abstract}
The rapid development of high speed railways urgently needs talents, and the affective commitment of railway employees has an important impact on the development of the enterprises. Based on the survey data of 462 railway employees in China, regression analysis was used to examine the influence of the perceived organizational support on overconfidence and affective commitment, and to examine the mediating effect of overconfidence between the perceived organizational support and affective commitment. The results showed that perceived organizational support has a significant impact on overconfidence and affective commitment, and overconfidence plays a part of mediating role between perceived organizational support and affective commitment.
\end{abstract}

\section{Introduction}

In recent years, great progress has been made in the technology and construction of Chinese highspeed railway multiple Units, which raises great challenges to the quality management of railway talents. As we all know, the development and progress of technology can not be separated from talents, and talent is the core competitiveness of an enterprise. Since the affective commitments of employees have an important impact on the talent retention, how to improve the railway employees' affective commitments and then make contributions to the development of railway industry has become a major research topic.

The perceived organizational supports are the degrees to which the employees feel the organizations pay attention to their contributions and care for their own welfare [1]. Affective commitments are the degrees to which employees identify and participate in the organization emotionally[2]. Previous studies have shown that the perceived organizational supports for college teachers are positively related to their affective commitments[3].

Overconfidence is derived from the research results of cognitive psychology. Overconfidence is a common psychological phenomenon, and it refers to an individual's overestimation of his ability compared to those of others[4]. As an important variable in the study of Psychology, Dong Xiaogang \& Wang Shunhong introduced the overconfidence into the field of organizational behavior management and studied the relationship between the perceived security risks, overconfidence and security performance[5]. However, few scholars studied the relationship between the perceived organizational support, overconfidence and affective commitment.

Based on the above analysis, combined with the existing theory of perceived organizational support and affective commitment, the psychological variable overconfidence is introduced to the organizational behavior management in this paper. In this paper, the Chinese railway employees are taken as the research object to explore the overconfidence in the mediating role of the perceived organizational support and affective commitment. We hope this paper can expand the research field of overconfidence, and provide the theoretical basis and empirical support for enterprises to improve their employees' affective commitments. 


\section{Theoretical Basis and Research Hypothesis}

\subsection{Influence of Perceived Organizational Support on Affective Commitment}

The perceived organizational support comes from whether the organization is willing to repay the employees' work inputs and meet their social emotional needs. High perceived organizational support means that employees are satisfied with the needs of recognition, respect, and social identity, and they will work harder. The formation of an employee's affective commitment requires a process. Firstly, employees will focus on the continuous commitment at the initial material level, and then pay attention to normative commitment at the institutional level, and finally the affective commitment at the spiritual level[6]. There is a close relationship between the corporate culture and social exchange, which has a significant impact on the affective commitment[7]. Research showed that the perceived organizational support, as a variable of the social exchange theory, had a significant positive impact on the affective commitment [8]. Therefore, there is the hypothesis H1:

H1: Perceived organizational support has a significant positive impact on the employee's affective commitment.

\subsection{Mediating Role of the Overconfidence Between Perceived Organizational Support and Affective Commitment}

The word overconfidence firstly appeared in the field of psychology. Wu Ruxin[9]believed that overconfidence was a potential corporate feature for all managers in a company. It might be a company culture, and this culture would make other managers of the company become overconfident, too. Some scholars have proved that the corporate culture has an impact on the overconfidence [10]. However, the perceived organizational support, as a variable in the corporate culture level, may have an impact on the overconfidence. The stronger the perceived organizational supports the employees feel, the more willing the employees are to dig out their skills and potentials and to show more flexibility in their work. At the same time, the employees' overconfidence level will also be improved. Therefore, the higher the employee's sense of perceived organizational support, the higher level of the employee's overconfidence.

Overconfidence, as an important variable in the field of psychology, has a certain influence on the affective commitment of the organization. In the context of Chinese collectivism, employees are proud of being fighting for enterprises. When employees' high level of self confidence is high, employees will think that their abilities are stronger than others. At the same time, they will also think that they do more contributions to the enterprise, and their employees' affective commitment to the organization becomes higher. Based on this, hypotheses H2- H4 are put forward:

$\mathrm{H} 2$ : The perceived organizational support has a significant positive impact on overconfidence.

H3: Overconfidence has a significant positive impact on the affective commitment of the organization.

H4: Overconfidence plays a mediating role between the perceived organizational support and organizational affective commitment.

\section{Research Objects and Methods}

\subsection{Research Objects}

A convenience sampling survey was conducted in 8 railway bureaus in China. A total of 600 questionnaires were sent out, and 540 were finally returned, of which 462 were valid, with an effective rate of $85.6 \%$. The descriptive statistics of the 462 valid questionnaires showed that $73.8 \%$ of the total number being male, 121 women, accounting for $26.2 \%$. 184 people were under the age of 30 to, accounting for $39.8 \%, 220$ people at the age of $31 \sim 40$ years old, accounting for $47.6 \%$. There were more than 58 people at the age of 41, accounting for $12.6 \%$. Among the interviewees, 252 people accounting for $54.5 \%$ were common staff, 172 middle and low level managers accounting for $37.2 \%$, 38 senior managers accounting for $8.2 \%$. 


\subsection{Measuring Method}

\subsubsection{The Measurement and Scoring Method of Overconfidence}

Based on Dong Xiaogang, Gao Ning \& Xia Xue [10], Glaser \& Weber [11], the overconfidence (OC) scale for railway workers, a total of 2 items, was made up by ourselves. For example, compared with your colleagues, if you do the same job, please estimate how many people have better "business skills" than you do. The results of the preliminary survey showed that the internal consistency coefficient (Cronbach's $\alpha$ ) of the scale was 0.873 and the reliability was good.

The item $O C_{1-2}$ adopts percentage scale. The main reason for the use of this scale is that the scale can be more finely answered than the Likert scale. For the convenience of calculation, the linear conversion is carried out by the following formula, and the range of $O C_{1-2}$ is $[-1,1]$.

$$
O C_{1-2}=\frac{50-x}{50}
$$

Where $O C_{1-2}$ is the calculation score for each item in the overconfidence scale. $x$ is the number the participant fills.

\subsubsection{The Measurement and Scoring Method of Perceived Organizational Support and Affective Commitment}

The perceived organizational support is measured by the Perceived Organizational Support (POS) scale which have 6 items[12]. For example, "the unit really takes care of my welfare." The measurement of affective commitment is based on the Affective Commitment (AC) scale[13]. The scale has 5 items, for example, "Even if the unit is poor, I'm not going to leave." Each item is measured by Likert 5 point scale. 1 means disagreement and 5 expresses a great agreement. The larger the number, the higher the perceived organizational support/affective commitment. In order to keep the range of the value of $P O S_{1-6} / A C_{1-5}$ consistent with the range of $O C_{1-2}$, the following formula is used for linear conversion.

$$
\operatorname{POS}_{1-6} / A C_{1-5}=\frac{x-3}{2}
$$

Where $P O S_{1-6} / A C_{1-5}$ is the calculation score of each item in the perceived organizational support / affective commitment scale. $x$ is the number the participant fills.

\section{Data Analysis}

\subsection{Reliability Test}

The internal consistency coefficient (Cronbach's $\alpha$ ) was used to test the reliability of 3 scales of perceived organizational support, overconfidence and affective commitment. The results showed that the internal consistency coefficients of all scales were all greater than 0.9 , indicating that all scales had good reliability.

\subsection{Validity Test}

The validity of each scale was tested by factor analysis. The results showed that the KMO values of the perceived organizational support scale and the affective commitment scale were all above 0.8 , and the KMO value of the overconfidence scale was 0.5 . The reason why the KMO value of the overconfidence scale was 0.5 was that overconfidence used only 2 items. According to the mathematical definition of the KMO test statistics, the KMO values of the 2 items were constant 0.5 . Therefore, the KMO value had no reference value and whether it was suitable for factor analysis was judged only according to the results of Bartlett sphericity test. The test values of the Bartlett sphericity of each scale were both significant at the significant level of 0.05 . Therefore, the 3 scales are suitable for factor analysis.

The factor analysis showed that there was only one factor with the eigenvalue value greater than 1 in each perceived organizational support, affective commitment and overconfidence scale. The load of each item in this factor was more than 0.8 , and the contribution rate of variance was higher than $70 \%$, which showed that the items had a high degree of explanation for the public factor, and the loss of information was less. So the result of factor analysis was ideal. 


\subsection{Correlation Analysis}

The results of pearson correlation analysis indicated that there was a positive correlation between perceived organizational support and overconfidence, perceived organizational support and affective commitment, and the correlation coefficients were 0.583 and 0.650 , respectively. There was also a positive correlation between overconfidence and affective commitment, and the correlation coefficient was 0.505 . Their correlation coefficients were both significant at a significant level of 0.001 .

\subsection{The Test of the Mediating Role of Overconfidence}

According to the testing method of mediating role[14], three steps were used to examine the mediating role of overconfidence between perceived organizational support and affective commitment. Known from Table 1, the first step was to examine the relationship between perceived organizational support and affective commitment. The results showed that the perceived organizational support had a significant positive effect on affective commitment. The regression coefficient, the direct total effect $\mathrm{C}$, was 0.292 , and it was significant at the level of 0.001 . So hypothesis $\mathrm{H} 1$ was verified. The second step was to examine the relationship between the perceived organizational support and overconfidence. The results showed that perceived organizational support had a significant positive effect on overconfidence, and its regression coefficient $\mathrm{A}$ was 0.584 , and $\mathrm{P}$ was less than 0.001 . So hypothesis $\mathrm{H} 2$ was verified. The third step was to examine whether there was a significant effect of perceived organizational support on affective commitment after controlling the influence of overconfidence (intermediary variables) on affective commitment. The results showed that overconfidence had a significant positive effect on affective commitment. Overconfidence played a part of significant mediating role in predicting the affective commitment by perceived organizational support. The corresponding regression coefficients B and C were 0.087 and 0.241 , respectively, and $\mathrm{P}$ values were all less than 0.001 . So $\mathrm{H} 3$ and $\mathrm{H} 4$ were verified. The mediating effect of overconfidence $\mathrm{A} * \mathrm{~B}$ was 0.051 , and the total effect $\mathrm{C}$ was 0.292 . The intermediate effect of overconfidence accounted for $17.4 \%$ of the total effect.

Table 1 The intermediate utility test of overconfidence between perceived organizational support and affective commitment

\begin{tabular}{|c|c|c|c|c|}
\hline Steps & Explanatory variables & Explained variables & \multicolumn{2}{|c|}{ Regression results $(\mathrm{N}=462)$} \\
\hline \multirow[b]{2}{*}{1} & Independent variables & Dependent variables & \multirow{2}{*}{\multicolumn{2}{|c|}{$\begin{array}{c}\mathrm{C}\left(\mathrm{S}_{\mathrm{C}}\right) \\
0.292^{* * *}(0.016)\end{array}$}} \\
\hline & Perceived organizational support & $\begin{array}{c}\text { Affective } \\
\text { commitment }\end{array}$ & & \\
\hline \multirow[t]{2}{*}{2} & Independent variables & $\begin{array}{l}\text { Mediating } \\
\text { variables }\end{array}$ & \multirow{2}{*}{\multicolumn{2}{|c|}{$\begin{array}{c}\mathrm{A}\left(\mathrm{S}_{\mathrm{A}}\right) \\
0.584^{* * *}(0.038)\end{array}$}} \\
\hline & Perceived organizational support & Overconfidence & & \\
\hline \multirow{2}{*}{3} & $\begin{array}{c}\text { Independent variables / Mediating } \\
\text { variables }\end{array}$ & Dependent variable & $\mathrm{B}\left(\mathrm{S}_{\mathrm{B}}\right)$ & $\mathrm{C}^{\prime}\left(\mathrm{S}_{\mathrm{C}}\right)$ \\
\hline & $\begin{array}{c}\text { Perceived organizational support / } \\
\text { Overconfidence }\end{array}$ & $\begin{array}{l}\text { Affective } \\
\text { commitment }\end{array}$ & $0.087^{* * *}(0.019)$ & $0.241^{* * *}(0.019)$ \\
\hline
\end{tabular}

*(1)A and $\mathrm{C}$ are the non-standardized regression coefficients of the perceived organizational support when the perceived organizational support predicts the overconfidence and affective commitment separately. $\mathrm{B}$ and $\mathrm{C}^{\prime}$ are the non-standardized regression coefficients of overconfidence and perceived organizational support respectively when they commonly predict the affective commitment. SA, SB, SC, SC' are the corresponding standard errors, respectively. (2) The results of multiple collinear test in the third step show that the highest value of the collinear VIF is 1.579 , and there is no multiple collinearity. (3) *expresses 0.05 significant levels. **expresses 0.01 significant levels. ***expresses 0.001 significant levels.

\section{Conclusion and Discussion}

Firstly, employees' perceived organizational support has a significant positive effect on affective commitment, which is consistent with previous studies. When employees feel a strong sense of perceived organizational support, employees' affective commitment to the organization will be 
deepened. In other words, the affective commitment of the employees will increase. At this moment, employees will work harder and contribute to the development of the enterprise.

Second, overconfidence plays a part of mediating role in the impact of perceived organizational support on affective commitment. Therefore, enterprises should give more support to employees in their career development, skills training and work process, and to improve employees' overconfidence level and employees' affective commitment to organizations, and then make employees contribute more to the development of enterprises.

In this paper, Chinese railway employees are taken as the research object. Chinese railway enterprise is a typical state-owned enterprise, so the enterprise culture and the culture of private enterprises are significantly different. Whether overconfidence is intermediary between perceived organizational support and affective commitment in the private can be studied in the future. And whether the nature of an enterprise is a disturbing variable between perceived organizational support and affective commitment can also be one of the research directions in the future.

\section{References}

[1]. Eisenberger, R., Huntington, R., Hutchison, S., et al. Perceived organizational support [J]. Journal of applied psychology. (1986) No. 71, p. 500-507.

[2]. Meyer, J. P., Allen, N. J. A three-component conceptualization of organizational commitment [J]. Human resource management review. Vol. 1 (1991) No.1, p. 61-89.

[3]. Hao Tianxia. A study on the relationship between perceived organizational support, organizational affective commitment and organizational citizenship behavior of College Teachers [J]. Journal of Northwest University (Philosophy and Social Sciences Edition). Vol. 41 (2011) No. 2, p. 173-175.

[4]. Svenson, O. Are we all less risky and more skillful than our fellow drivers? [J]. Acta Psychologica. Vol. 47 (1981) No. 2, p. 143-148.

[5]. Dong Xiaogang, Wang Shunhong. Research on relationship between perceived safety risk, overconfidence and safety performance [J].China safety science journal. Vol. 24 (2014) No. 9, p. 8-14.

[6]. Zhu Aiqin. Analyzing of organizational commitment formation process and strategies [J]. Finance \& Economics of Xinjiang. (2008) No. 5, p. 86-89.

[7]. Liu Xiaoping, Wang Chongming. Organizational commitment and its formation under the Chinese and Western cultural background [J].Foreign Economies \& Management. Vol. 24 (2002) No. 1, p. 17-21.

[8]. Song Li, Gu Jibao \& Yang Li. An empirical study of the impact of human resource practice on employees' perceived organizational support and organizational commitment [J].Science and technology management research. (2006) No. 7, p. 157-160.

[9]. Wu Ruxin. The effects of top managerial overconfidence on the corporate investment decision [D].Changsha: Central south university, 2011.

[10]. Dong Xiaogang, Gao Ning \& Xia Xue. The impact of safety culture on safety performance in construction enterprises [J].Highway. (2014) No. 8, p. 140-146.

[11]. Glaser,M., Weber, M.. Overconfidence and Trading Volume [J].Geneva Risk and Insurance Review.Vol.32 (2007) No. 1, p. 1-36.

[12]. Su Wensheng. HR empiricism of public institution based on POS [D].Chengdu: southwest jiaotong university, 2011. 
[13]. Ling Wenquan, Zhang Zhican \& Fang Liluo. The research on the structure model of Chinese employee's organizational commitment [J].Journal of management sciences in China. Vol. 3 (2000) No. 2, p. 76-81.

[14]. Wen Zhonglin, Hou Jietai \& Zhang Lei. A comparison of moderator and mediator and their applications [J]. Acta psychologica sinica. Vol. 37 (2005) No. 2, p. 268-274. 